\title{
On the innovation and development of embedded systems from the perspective of environmental science and technology aesthetics
}

\author{
YUE Xiangyu ${ }^{1}$, YUE Youxi ${ }^{2}$ \\ ${ }^{1}$ School of Management and Engineering, Nanjing University; Nanjing Jiangsu; 210039; PR China \\ ${ }^{2}$ School of Chinese Literature and Journalism, Shandong University of Technology; Zibo Shandong; 255049; PR China
}

\section{Email address:}

yuexiangyu168@gmail.com(YUE Xiang-yu),youxiyue@aliyun.com(YUE You-xi)

\section{To cite this article:}

YUE Xiangyu, YUE Youxi. On the Innovation and Development of Embedded Systems from the Perspective of Environmental Science and Technology Aesthetics. Journal Electrical and Electronic Engineering. Vol. 1, No. 4, 2013, pp. 68-72.

doi: 10.11648/j.jeee.20130104.11

\begin{abstract}
The environmental science and technology aesthetics is a new interdisciplinary application aesthetic subject, which arises with the inspiration of contemporary ecological concepts, environmental ideas, and aesthetic thoughts. It is based on the theories of ecological ontology, ecological ethics and ecological value, and it is the perfect combination of science and technology and environmental aesthetics, and it is the science of the general laws in which science and technology are studied, innovated and developed in accordance with the principles of environmental aesthetics. The embedded system is a "dedicated computer system embedded in the object system", and it is technology-intensive, investment-intensive, highly decentralized and continuously innovative knowledge-intensive system. The embedded system innovation has the close and indivisible relationship with the environmental science and technology aesthetics: The environmental science and technology aesthetics is instructive to the embedded system innovation; while the embedded system innovation is concrete practice and application of the environmental science and technology aesthetics theory. The environmental science and technology aesthetics has got rid of the Western traditional dualistic and opposing thinking mode, surpassed the modern tool reason and anthropocentrism, and promoted the environmental development of embedded system. The environmental science and technology aesthetics is the important theoretical resource that guides the environmental development of embedded system, and an important guarantee that makes the embedded system develop and innovate along healthy, scientific, environmental road. The environmental embedded system must have broad development prospects.
\end{abstract}

Keywords: Environmental Science and Technology Aesthetics, Embedded System, Innovation, Development

\section{Introduction}

The rapid development of modern science and technology has greatly changed the modern human life and brought great benefits to our life and work. But many scholars think, it is like a double-edged sword, while bringing the facility to mankind, also stabbed human. ${ }^{[1](\mathrm{P} 1)}$ For example, it caused the alienation of human nature seriously, tremendous damage to the natural ecological environment, and made the human spirit into the state of alienation bewilderment and confusion, etc. Some people even believe that more advanced technology, greater alienation to human life. Therefore, as a representative of modern high-tech, "embedded" system is bound to cause the most, the largest and even immeasurable alienation to human life. However, in the author's opinion, the embedded system is a highly developed science and technology, but it can't be an alarmist, because as a tool of science and technology it cannot bring human alienation, but the use of science and technology of human beings. More fundamentally, is the human survival in the "cultural" root that caused the human alienation. Therefore, in order to prevent the alienation of the embedded system to human beings, first of all is to adjust the human overall survival strategy, to change human thinking in the cultural choice actively, thus completely change the status of human. This cultural choice is the ecological culture which is the core of ecological aesthetics. Ecological aesthetics is a cultural and scientific panoramic concept system that includes man and nature, man and society, man and man and all motion system. Only the perfectly combination of aesthetics and embedded system innovation onto the 
"ecological technology aesthetics" innovation road can eliminate the alienation of embedded system caused fundamentally, change and improve the living circumstances of human beings. Therefore, the ecological technology aesthetics has important theoretical significance and practical value for "embedded" system innovation, development and perfection.

\section{The Basic Connotation of Environmental Science and Technology Aesthetics}

To understand the basic connotation of environmental science and technology aesthetics, we must first understand the concept of "Ecological Aesthetics." Ecological Aesthetics is an interdisciplinary science based on the ecology and aesthetics. With the integral thinking of the ecosystem, ecology breaks the thinking tradition of subject-object dichotomy from the West, and views human race as a part of nature, thinking about in holistic nature, how humans develop in harmony with nature. Ecological Aesthetics is a new form of aesthetic theory which originates from the combination of ecology and aesthetics. It investigates aesthetic issues using ecological theory and method, important aspects of the ecology are absorbed into the aesthetic studies, and ecological way of thinking is applied to the field of aesthetics, thereby establishing the new form of aesthetic theory which adapts to social development. Its uniqueness lies in the organic integral way to look at aesthetic issues. Ecological beauty is the presentation of the aesthetic significance, it is realized by maintaining the ecological balance of the ecosystem of the entire world, including nature and human society, it suggests that man and nature have achieved a natural aesthetic state of coordinated development as well as coexistence and co-prosperity.

Environmental science and technology aesthetics mainly refers to the science arising from the combination of ecology, aesthetics and science and technology, it is an emerging interdisciplinary new aesthetic applications discipline which engenders with the inspiration of contemporary ecological concepts, environmental concepts and aesthetic concepts. Its theoretical foundation is based on Ecological Theory ${ }^{[2]}$ (P52), Ecological Ethics ${ }^{[2](\mathrm{P} 105)}$ and Ecological Value ${ }^{[2](\mathrm{P} 76)}$. It is specific application of theoretical principles of ecological aesthetics in the areas of technological innovation, which means perfect combination of science and technology and ecological technology aesthetics, making the research, innovation and development of science and technology be in accordance with the principles of ecological aesthetics. It is the science which investigates the ecological phenomenon ecological relationships and ecological laws of science and technology as well as the nature, from the aspect of aesthetic with the aesthetic attitude, viewpoint and method. Ecological technology aesthetic is the concrete embodiment of ecological ontology and aesthetic in the technology sector.
It has great innovation and pioneering. Ecological technology aesthetics not only focuses on qualitative (i.e., the nature of existence) research of technology and nature, reconstruct the philosophical foundation during the inspection of inner life essence of science as well as technology and nature, and thus falls within the physical properties of category; but also comes with more aesthetic meaning, focusing on external form (namely, the existence of appearance) of science and technology and nature, and thus falls within the scope of phenomenology.

At the same time, it puts more emphasis on the real relationship of survival between science and nature, science and people, as well as nature and people. More specifically, it centers on natural ecology, orients the value of science and technology, and reconstructs reality. Environmental science and technology aesthetics covers 3 dimensions of peoples' aesthetic concern toward science and technology, i.e., to take truth, goodness and beauty respectively for beauty, and the three concrete means of which lie in the reinterpretation of science and technology by intellect, the concern by sensibility, and the recreation by human will. Environmental science and technology aesthetics is an important theoretical source for directing the modern technological development, which abandons the traditional thinking pattern of dichotomous opposition, transcends modern instrumental rationality and anthropocentrism, and promotes the ecological progress of modern science and technology. Its significance lies in maintaining the soundness and the benevolent development of modern science and technology, improving the construction of socialist eco-civilization and building a beautiful China, even a beautiful world.

\section{Environmental Science and Technology Aesthetics and the Innovation of the Embedded System}

The so-called "embedded" system is the professional computing system that is embedded into the object system, and that is system devices used to control, monitor, or assist the operation of equipment, machinery or plants. "Embedding", "specificity" and "computing system" are the three basic elements of embedded system. The embedded system is a complex of software and hardware and can contain attendant devices such as machinery. Now a definition for the system prevalently approved is a professional computing system centering around application, based on computing technology, the software and hardware of which can be reduced and catering to the strict demands made by the system of application for its function, reliability, prime cost, volume and power dissipation. The embedded system (ES) is a renewed product replacing older ones combining advanced technology including computing technology, communication technology, semi-conductor technology, microelectronic technology, technology of transmitting audio and visual data with concrete object of application. Therefore, it is intensively technical, involving 
huge investment, highly scattered and increasingly original intellect-intensive system. There is an inalienable intimacy between innovation of the embedded system and environmental science and technology aesthetics: the latter provides guidance for the innovation of the former; whereas the former serves as a specific practice of the theory behind the latter.

Eco-technological aesthetics was instructive to the embedded system innovation, because they have the intrinsic unity. As we know, the philosophical foundation of ecological aesthetics is the science of ecology existing theory, it inherits the characteristics of system theory, emphasizing ecological existence not human, social and other kinds of natural things scattered, but the overall existence. It is in between the various elements of the affirmation of human, social and natural things interact to form ecological existence on an overall basis, negative ecological overall equal to each part of the things and people simply the sum of the mechanical concept, adhere to the ecological system has its own specific matter, by the people, society and the nature of things, nonlinear interaction role in the formation of organic whole between intrinsic factors; special ecosystem exists on a higher level of ecosystem environment, under the influence of a higher level of ecological law, status, development trend of the whole, provisions characteristics of this ecological system is from the system environment to obtain qualitative. In addition, the ecological ecology existing theory emphasized human, social and natural existence is organic, they are organic whole system. It is starting from the ecological concept of science, organic ecological existence certainly people, society and natural things, and the organic life, with their own ecosystem self-organization, self-regulation, self-choice ability, put the whole world portrayed by human, society and nature, mutual penetration, interaction coordinate with each other, the evolution of the organic unity. [2] (P52) ecological existence of the integrity and systematic, coincided with the embedded system innovation is unified. Embedded system has inherited the overall characteristic of system theory, deny the existence of the whole is equal to the sum of each part of things simple mechanical concept, emphasizing its control, monitoring or auxiliary equipment, machinery and equipment operation system elements and the elements of the whole is greater than the simple sum of the complex, and the simple sum of its software and hardware has more than two. The computer technology, communication technology, semiconductor technology, microelectronic technology, speech and image data transmission technology, as well as sensors and other advanced technologies, combined into a technology and knowledge intensive system. Ecology existing theory thought, ecological, social and natural things is the existence of process. From the perspective of self-organization theory, the existence of faithfully described as relational, procedural and substantive of unity, the natural ecological processes is a self-organized transport process unified, adhere to the "entity" of different levels, different levels of the connections between things are in the same transport process

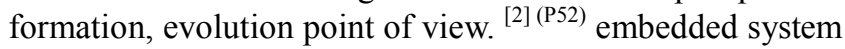
is also a system, embedded system innovation also has the process, is also a process of organization. The innovation and development of embedded system is also different levels of "existence", different levels of the connections between things in the unified operation during the formation and evolution process.

Eco-technological aesthetics is a new theory based on ecological science and the complexity science theory, is the summary and sublimation of science theory It negated the traditional metaphysics ontology, and discards and returns ancient organic holism, in a higher level. "Ecological Existentialism" including the ontology sense of philosophy and the view of nature, is the philosophical foundation of ecological aesthetics, and the inevitable product of the spirit of the times. Ecological science and technology aesthetics is the specific application of ecological civilization in the field of social practice. Based on the ecological ontology, Eco-technological aesthetics as a new aesthetic paradigm is the natural humanization and human nature of the organic unity of the new scientific aesthetic paradigm. ${ }^{[2] \text { (P52) }}$ Eco-technological aesthetics and embedded system innovation have its unity, ecological science and technology aesthetics has important theoretical guiding significance on embedded system mechanism innovation, function innovation. And the embedded system innovation is a concrete practice of ecological science and technology aesthetics theory in the field of science and technology innovation.

\section{Development and Prospects of "Embedded" Eco-Technology}

Embedded system is a special computer system which embedded in to the object system. Its development has experienced three stages: the first stage, embedded system also in its early stages is dedicated computer to function relatively simple or single chip microcomputer as the core of the programmable controller. The second stage, the embedded system is based on embedded CPU and embedded operating system as a symbol. The third stage, the embedded system on chip technology and Internet technology as a symbol. Embedded system plays an important role in the field of computer application with the characteristics of flexibility, strong specificity, good real-time performance, strong anti-interference ability, scalable, high reliability, low power consumption and large capacity of network. Therefore, the embedded system becomes one of the most popular in the most promising areas of IT applications. Now, micro sensors with sensing, computing and communication capabilities are developed which people use communication, embedded computing and sensor technique. People can use the wireless sensor network at any time and any place, under any condition to live monitoring, sensing, collecting collaboration network 
coverage area of a variety of environmental or monitoring information, from information processing which can get detailed and accurate information and finally delivered to users who need this information. Therefore, the embedded systems are widely used in military, national security, environmental monitoring, aerospace, industrial control, traffic management, health care, manufacturing, combating terrorism, information household appliances, home intelligent management system, POS network, e-commerce and other fields. Especially with the development of intelligent home appliances, embedded system is more and more important. For example, the mobile phone, PDA, electronic dictionary, video phone, digital camera, VCD/DVD/MP3 Player (DC), digital camera (DV), U-Disk, the set-top box (Set Top Box), high definition television (HDTV), game machine, intelligent toys, switch, router, $\mathrm{CNC}$ equipment or instrument, automobile electronic, electrical control system, medical equipment and so on, are the typical embedded system we used.

In this information and intellectual economy times, the development of computer science and technology is deeply into all aspects of human life. Especially the high performance distributed computing standard tools such as high performance multi-core processor, optical fiber and Myrinet high-speed network, has greatly promoted the cluster is calculated by "high performance computing" to "high performance computing" transformation. However, how to manage large collections of resources scheduling? How to give full play to each member of the working capacity and reduce the energy consumption of the system? How to make the embedded system on innovation of ecological technology and green development road? These are problems need to solve. How to make the computation and communication is omnipresent and ordinary users can easily enjoy the service? Many research fields across the mobile computing, embedded system, natural human-computer interaction, software structure of pervasive computing technology is playing an important role. The harmonious relationship between the embedded system and natural, human being, and society, has become a very important problem in embedded system innovation must be considered. To sum up, the development trend of embedded system mainly includes the following points: firstly, the development and innovation of the embedded system, must develop along the ecological technology aesthetics road, or take the ecology of embedded system technology development. Secondly, we need to innovate and develop the embedded hardware platform for the system, and to formulate industry standards. Thirdly, with the innovation and development of related technology, SoC will become a mainstream application. Fourthly, the development tools and the embedded operating system with high innovation and the development which encourage and support the application of embedded system software development are becoming more and more complex. Fifth, innovation and development for network interconnection of the embedded system will become the development trend of the field. Sixth, the embedded system is on the way to the new direction of the development of embedded computing model. The new embedded system Includes: support graphics, human-computer interaction and interactive nature, or multimedia embedded man-machine interface lead to users can simple use the operation without learn; embedded system to support the development of programmable, like using embedded JAVA technology, can be dynamically loaded software upgrades; support distributed computation with other embedded system or computer system interconnection, distributed computing environment.

The innovation and development of embedded system must take the "ecological technology innovation" road which has become the requirements of the times and the trend of development. It is switched the innovation of science and technology from the simple pursuit of economic benefits to human life in the future, sustainable development and green peace research. Ecology Embedded system will be a kind of carrier that makes the person and nature closer to each other. We can through its form and function to realize the design aesthetic and creation of ecological technology which the philosophy, namely technology and ecological technology aesthetics complement each other. The natural beauty of the ecological technology is not a general sense, natural beauty is the aesthetic value of the nature itself, while the ecological technology beauty is the highlight of natural ecological value in its innovation in science and technology. Embedded system through ecological aesthetic appearance, we can whole view its inherent nature. Ecological embedded system the inherent pristine nature will become the human emotional support "sampans". It is loaded with human' passions, it is the communion between human and ecological embedded system, rather than ecological communion with nature. Behind the natural ecology of the embedded system, which is the ecological idea of man and nature to obey, reflects the close relationship between man and nature live in harmony.

Future technological innovation and development will inevitably follow the environmental science and technology aesthetics which is also the only way. Ecological science and technology innovation, whether it is the choice of materials, technology products, external form, or its internal function and structure will reflect the aesthetic character of the natural ecology. Even in the beginning of product design, it is necessary to take into account the issue of recycling products: either recycled either non-polluting in nature digestion. Ecological and technological innovation, not only to consider the economic benefits of the product, but also should consider the social and ecological benefits of products, attention to their natural attributes and ecological aesthetic properties. In modeling Eco-technology products take its natural aesthetic attributes and ecological characteristics into considering, while people using the product, you can enjoy the natural beauty and sentiment; in the psychological and emotional desires, it ignite the desire and protection for natural ecology. The innovation and development of this eco-technology system is following a 
green technology path which meets human ecological aesthetic. Only then will it not give greater pressure environment, humans can only be in harmony with nature, only in this high speed, low-carbon society without losing our lives, will truly benefit our children and grandchildren.

\section{Conclusion}

In summary, ecological science aesthetics is a new interdisciplinary aesthetic application discipline arising under an inspiration of contemporary ecological concepts, environmental ideas, and inspiration aesthetic concepts arising under an emerging interdisciplinary application of new aesthetic disciplines. Its existence of ecology, ecological ethics and ecological value theory as the theoretical basis, is a combination of science technology and ecological aesthetic perfection, general rules of research, innovation and development in accordance with the principle of ecological aesthetics to science and technology science. It is from the angle of aesthetics, aesthetic attitude, viewpoint and method, ecological phenomena, research of the technology and human and natural ecology and ecological law science. Embedded system is "to the object system embedded in a special computer system", also refers to the control device, system monitor or auxiliary equipment, machinery and equipment operation. Embedded system (ES) is the computer technology, communication technology, semiconductor technology; microelectronic technology, speech and image data transmission technology, products and sensors and other advanced technology and concrete application object combining update. Therefore, it is a knowledge-intensive technologies, investment intensity, high dispersion, continuous innovation. Embedded system innovation and ecological technology aesthetics have close relationship: the ecological technology aesthetics has the guidance of embedded system and embedded system innovation; innovation is the specific practice and application of ecological technology aesthetics theory. Ecological science and technology aesthetics from two opposite thinking mode of the Western tradition, beyond the modern instrumental rationality, transcendence of anthropocentrism, and promote the ecological development of embedded system. It is to keep the embedded system health, scientific development, has the vital significance. Ecological science and technology aesthetics is an important theory of ecological resources to guide the development of embedded system, is an important guarantee for the development and innovation of embedded system development along a healthy, scientific, ecological road.
Innovation and development will ecological embedded system has broad prospects for development.

\section{Introduction to the Authors}

YueXiangyu (1992- ), who is a male, Han nationality, was born in Weifang, Shandong, with BA, Nanjing University, mainly engaged in electrical information and automation, aesthetics and other aspects of learning and research, specializing in modern industrial embedded control systems, networked control systems, intelligent control. He presided over one national college student science and technology innovation project, taking part in the research on one "Eleventh Five-Year Plan" education and science key project of the Education Ministry, and one soft science research project of Shandong Province. He has 12 science and technology papers published in Chinese and oversea academic journals. He has won the 1st scholarship of China, the 1st scholarship of people, the 1st and the 2nd prizes of China Education Robotics Competition, the 1st prize of China Mathematical Modeling Contest, the top award of scientific research achievement of Nanjing University, two China science and technology patents and the honorary title, "three-good-student" of Jiangsu Province. In addition, as a representative of Nanjing University, he went to the National University of Singapore to participate in an academic exchange program. Address: School of Management and Engineering, Gulou Campus of Nanjing University, 22, Hankou Avenue, Gulou District, Nanjing, Jiangsu Province, PR China; Zip: 210039.

YueYouxi, male, Han nationality, post-doctor, visiting scholar of Harvard University, was born in Weifang Shandong province in June, 1967, who is a professor, graduate student tutor of the School of Chinese Literature and Journalism, Shandong University of Technology \&. Shandong Research Base for the Soft Sciences of Eco-culture and Recycling Economy, and principally good at teaching and doing research on the literature theories, aesthetics, environmental culture, and so on.

\section{References}

[1] YueYouxi. Pursuing Poetic Inhabitation [M].Beijing: People's Publishing House2009.

[2] Yue You-xi. The Environmental Aesthetics [M]. Beijing: People's Publishing House, 2007.

[3] $\mathrm{Hu}$ Jintao,the Chinese Communist Partyeighteenreport[A],XinhuaNovember 8, 2012. 